\title{
LXIII. On the theory of storms, with reference to the views of Mr. Redfield
}

\section{Robert Hare M.D.}

To cite this article: Robert Hare M.D. (1841) LXIII. On the theory of storms, with reference to the views of Mr. Redfield, Philosophical Magazine Series 3, 19:126, 423-432, DOI:

10.1080/14786444108650461

To link to this article: http://dx.doi.org/10.1080/14786444108650461

册 Published online: 01 Jun 2009.

Submit your article to this journal

Џ Article views: 2

Q View related articles $₫$ 
LXIII. On the Theory of Storms, with reference to the Views of Mr. Redfield. By Robert Hare, M.D., Member of the American Philosophical Society, Professor of Chemistry in the University of Pennsylvania*.

1. M R. REDFIELD'S idea, that tornadoes and hurrilities.

$1 \mathrm{I}$ canes are all whirlwinds, involves some improbabi-

2. It requires that during every hurricane there should be blasts of nearly equal force coinciding with every tangent which can be applied to a circle. Thirty-two ships, equidistant from the axis of gyration and from each other, should each have the wind from a different point of the compass with nearly equal force. The only modification of which this view of the case admits, is that resulting from the progressive motion which tends to increase the velocity of the wind on the side on which this motion concurs with that of the whirl, and to produce upon the opposite side a corresponding diminution. Moreover, as respects any one station, the chance would be extremely unfavourable that the same hurricane should twice proceed from the same quarter: and yet in the course of time it would be felt, at any station, to proceed from many different directions, if not from every point of the compass.

3. Mr. Redfield has alleged, that he observed proofs of gyration in the effects of the New Brunswick tornado; but I think that the survey of Bache and Espy shows that it would not be consistent with the facts to suppose such a motion, unless contingently; and that it could only be a casual effect of the currents rushing towards the axis of the tornado $\dagger$.

4. Being of opinion that calorific expansion is inadequate to explain the afflux of wind towards the equator, it is alleged by Mr. Redfield, "that the space previously occupied by the atmosphere so left behind, is, by the centrifugal action of the earth's rotation, constantly supplied from higher latitudes."

5. I presume that the meaning of this allegation is, that the centrifugal force communicated to the air at the equator by the diurnal revolution of the earth, lessening the gravity of the air thus affected, causes it to rise, and give place to those portions of the atmosphere which, existing where the diameter of the earth is less, have less rotary motion. Admitting an afflux to arise in this way, could it have any other effect than that of accumulating air over the equator, compensating by quantity and altitude for the loss of weight arising from a greater centrifugal force pertaining to that region? But, on

* Communicated by the Author.

+ See fifth volume of the American Philosophical Transactione. 
the other hand, if we attribute the ascent of the air at the equator to heat, the theory of calorific circulation will account for the continuance of the process.

6 . In ascribing the prevalence of westerly winds in the upper regions of the atmosphere to the deflection of the trade winds by our mountains, Mr. Redfield's explanation harmonizes with the theory of Halley. In fact, is it not reasonable, that, as the water accumulated by these winds in the Gulf of Mexico is productive of a gulf-stream, there should be an aërial accumulation and current corresponding with that of the aqueous current, which is designated by the name above-mentioned?

7. But not perceiving that the trade winds cannot be explained without the agency of temperature, $\mathrm{Mr}$. Redfield, in the following paragraph, rejects the influence of heat:-

8. "To me it appears that the causes of the great storms may be considered. to indicate with entire certainty the great law of circulation in our atmosphere; and that the longcherished theory, which is founded on calorific rarefaction, must give place to a more natural system of winds and storms, founded mainly upon the more simple conditions of the great laws of gravitation."

9. It would seem from this paragraph as well as others, that Mr. Redfield admits of no other cause of atmospheric currents besides that of gravitation. But in the absence of calorific and electrical reaction, what other effect could gravitation have, unless that of producing a perfect state of inert quiescence?

10. It is remarkable that the author, after ascribing the trade winds to momentum, as the antagonist of gravitation, loses sight of it in this summing up of the causes of atmospheric currents!

11. If, as $\mathrm{Mr}$. Redfield alleges, the minuteness of the altitude of the atmosphere, when compared with its horizontal extent, be an objection to any available currents being induced by calorific rarefaction, wherefore, for the same reason, should not momentum, or any other cause diminishing or counteracting the influence of his chosen agent, gravity, be equally inefficient?

12. Assuming that the motion of the air in hurricanes is always gyratory, Mr. Redfield considers gyration as a cause of these terrible meteors! How far his language on this subject is reasonable or consistent, may be seen from the following paragraph, which I quote from one of his essays, published in Silliman's Journal for 1834, vol. xxv. page 125:-

13. "Notwithstanding these general and determinate ho- 
rizontal movements, the equal distribution of the atmosphere over the surface of the globe, which results from gravitation, tends to prevent any very rapid or violent motion in any specific direction, and consequently to prevent violent and destructive winds. But owing to the tendency of all fluid matter to run into whirls or circuits, when subject to the influence of unequal or opposing forces, a rotative movement of unmeasured violence is sometimes produced. This peculiar movement, which in its most active state is sometimes distinguished by the name of tornado or hurricane, assumes every possible variety of position, appearance, velocity and extent, and is the only known cause of violent and destructive winds or tempests."

14. Agreeably to this paragraph, gravitation, in lieu of being, as previously alleged, the main basis of winds and storms, tends to produce that equal distribution of the atmosphere over the surface of the globe, on which $I$ have insisted.

15. But if neither gravity, nor calorific expansion, nor electricity be the cause of winds, by what are they produced?

16. He alleges that the fluid matter has a tendency to run into whirls or circuits, when subject to the influence of unequal or opposing forces, and that in this way a rotative movement of unmeasured violence is sometimes produced.

17. If this were true, evidently whirlpools, or vortices of some kind, ought to be as frequent in the ocean, as, agreeably to his observation, they are found to be in the atmosphere. The aquatic gulf-stream, resulting from the impetus of the trade winds, ought to produce as many vortices in its course as the aerial currents derived from the same source, especially as in the ocean the great laws of gravitation have full liberty to act without any important interference from calorific changes, to which the advocates of the agency of such changes in producing wind will not ascribe much efficaicy, where non-elastic liquids are in question.

18. There are few vortices or whirlpools in the ocean, because there are in few cases descending currents towards which the surrounding waters are concentrated. Of course, vertical currents cannot arise from any imaginable cause.

19. The conflicts of "opposing or unequal forces" do not produce curvilinear motion, unless there be a successive deflection, as in the case where it results from centripetal force, or the influence of gravity upon a projectile. If one of two directly opposite forces be less than the other, retardation will ensue, and a lateral current or currents carrying off the excess of momentum. If currents encounter each other obliquely, a diagonal current will result. I doubt if a whirlpool ever 
takes place without a centripetal force resulting from an hiatus.

20. But the author has not informed us how these unequal or opposing forces are generated in the atmosphere. Without any assigned cause, he appeals to " certain opposite and unequal forces by which a rotative movement of unmeasured violence is produced." This rotative movement, although alleged, as above, to be an effect, is stated immediately afterwards to be "the only known cause of violent and destructive winds or tempests."

21. In a memoir on the causes of tornadoes and water-spouts, and in some subsequent communications published in the Transactions of the American Philosophical Society, and republished in Silliman's Journal, various facts and arguments were mentioned, tending to prove that the proximate cause of the phænomena of a tornado is an ascending current of air, and the afflux of wind from all points of the compass to supply the deficiency thus created.

22. In this mode of viewing the phænomena, no difference of opinion exists between Bache and Espy and myself, however we may differ respecting the cause of the diminution of atmospheric pressure within the track of a tornado, which gives rise to the ascending current.

23. I adduced several facts, upon the authority of the skilful survey made by those gentlemen, proving that the effects were in some cases inconsistent with the existence of a whirl; and I mentioned one which conld not be explained without attributing it to a gyratory force. I was led to consider gyration as a contingent, not an essential feature in the meteors in question.

24. It appeared reasonable to suppose that the confliction of confluent streams of air rushing towards an axis moving progressively, might be productive of a whirling motion. The contortion of six feet of the upper part of a brick chimney upon the lower portion, so as to cause the corners of either portion to project over the sides of the other, was deemed inexplicable without ascribing it to gyratory force. Subsequently, however, it occurred to me that this fact was more likely to be the result of a local than of a general whirl; since in the latter case, the chimney could not have been twisted as described, without being precisely at the centre of the whirlwind. That such could have been its position, appears to me extremely improbable; and had it been so situated, as the whirlwind was estimated to be moving progressively at the rate of seventeen miles per hour, it is to me incomprehensible how the portion which was dislocated could have escaped an overthrow. Evidently, although twisted upon its base while 
concentric with the gyration, it would in one second of time have been twenty feet upon the windward side of it, consequently subject to the tangential force of the whirlwind. I adduced this, as well as other facts, to prove that in torna. does and hurricanes there are local whirls, causing bodies which are of a nature to favour electrical discharge to be particularly affected ; a fact which is admitted by Mr. Redfield, was considered by Espy and Bache, as well as myself, to be irreconcilable with the idea that a general whirling motion is essential to tornadoes. I allude to the circumstance, that when several trees were prostrated one upon the other, the uppermost was found to have fallen with the top directed towards the point towards which the meteor was moving; while the direction in which the lowermost trees were found to have fallen indicated that they were overthrown by a force in a direction precisely the opposite of that which had operated upon those above-mentioned.

25. Mr. Redfield has not made any effort to show how the trees could have been piled upon each other, as described, but, on the contrary, admits that a whirlwind would blow oppositely, on opposite margins of the whirl. As this appears to me quite evident, I cannot understand how the opposite forces belonging respectively to the different sides of the whirlwind, can be made to bear successively upon one spot, so as to cause trees to fall in diametrically opposite directions.

26. Another fact, irreconcilable with a general whirling motion, was adduced 'by Messrs. Espy and Bache. One of the four posts, upon which a frame building was supported, was first moved towards the tornado, as it advanced; in the next place as it moved away, so as to make two furrows in the ground. In the interim the frame was protected by a larger building, which intervened between it and the tornado. I am utterly unable to understand how the transient tangential forces of a whirl wind blowing oppositely, on the opposite margins of its track, could thus move the post in question, so as to make two distinct furrows in the ground indicating two successive impulses, in directions of which one was at right angles with the other. Mr. Redfield admits that " the confused directions of fallen bodies is distinctly recognized by all the parties to this inquiry." Conceding that amid this confusion he has been enabled, by a survey, to show that the directions in which certain trees fell are consistent with their having been subjected to a whirlwind, it does not demonstrate gyration to be an essential feature of tornadoes. It is sufficiently accounted for by considering it as a fortuitous consequence of the conflict of currents rushing into a rarefied vortex. 
27. Mr. Redfield adopts the singular determination of not noticing the "insuperable difficulties" of the hypothesis which he has undertaken to set aside. The advocates of the disputed hypothesis are not aware of any such difficulties: is it correct to allege their existence without mentioning the facts and arguments which justify this allegation? Without repeating here the evidence and the reasoning which $I$ have already published on this subject, I will advert to one fact which is utterly irreconcilable with Mr. Redfield's " rotary theory." I allude to the statement of a most respectable witness, that while the tornado at Providence was crossing the river, the water, which had risen up as if boiling within a circle of about 300 feet, subsided as often as a flash of lightning took place. Now supposing the water to have risen by a deficit of pressure resulting from the centrifugal force of a whirl, how could an electrical discharge cause it to subside?

28. I have already, I trust, sufficiently shown that the abortive explanation which $\mathrm{M}$. Redfield dignifies with the title of " his theory of rotary storms," amounts to no more than this : that certain imaginary, nondescript, unequal and opposing forces produce atmospheric gyration; that these gyrations, by their consequent centrifugal force, create about the axis of motion a deficit of pressure; and hence the upward force displayed by tornadoes and hurricanes. I cannot give to this alleged theory the smallest importance, while the unequal and opposing forces upon which it is built remain in perfect obscurity, the author having disclaimed both the agency of heat and electricity.

29. But admitting a whirlwind to be produced, not by a deficit of pressure about the axis, but by unequal and opposing forces acting externally in any competent way whatever, it is perfectly evident that any deficit of pressure about the axis consequent to the resulting centrifugal force, could only cause a descending aërial current, while it could not tend in the slightest degree to carry solids or liquids aloft.

30. It must be obvious that the stratum of air on the earth's surface, partaking of the circular motion, must also partake of the centrifugal momentum, and of course would have a disposition the very inverse of that which would cause them to rush towards the axis; while heavier bodies being surrounded by the rarer mediums consequent to the whirl, would have their gravity less counteracted than usual. I cannot help thinking, that as respects the application of his " rotary theory" to explain tornadoes, these arguments will amount to a "reductio ad absurdum."

31. Mr. Redfield infers that the whirlwinds, of which he as- 
sumes the existence, have a property which he alleges to be observable in "all narrow and violent vortices, viz. a spirally involute motion quickened in its gyrations as it approaches towards the centre of the axis or whirl."

32. But is it not evident, that if any mass of matter be made to revolve by unequal and opposing forces, or by any other than those resulting from the centripetal force, caused, as already described, by an ascending current, the gyration will not quicken in proportion as the gyrating matter may be nearer the centre, but on the contrary will be slower as the distance from the axis may be less? It appears to me, that the only case in which gyration is found to quicken in proportion as the matter involved approaches the vortex, is that which results from the confluence caused by an ascending or descending concentric current. So far therefore as Mr. Redfeld's observations confirm the idea that the whirling motion in tornadoes quickens towards the centre, it tends to confirm the opinions which he combats, and to refute those which he upholds.

33. To conclude whether or not the efforts which I have made, to show that the phænomena of tornadoes and hurricanes arise from an electrical discharge by convection, be justifiable, I think it will be conceded that any theory of storms which overlouks the part performed by electricity must be extremely defective.

34. Both by Messrs. Espy and Redfield the influence of this agent in the phænomena of nature is entirely disregarded, although with the storms, which have been especially the subject of their lucubrations, thunder, lightning, and convective discharge are most strikingly associated.

35. I will conclude with subjoining the following propositions, inferences, or allegations; which are so evident to my mind, that $\mathrm{I}$ am at a loss to understand that they have not had a similar influence upon the minds of all who are conversant with the science of electricity.

36. Our experiments make us familiar with two processes of electrical discharge. In one of these electricity passes in the form of sparks or flashes; in the other it may be conveyed without any perceptible evolution of light, by the alternate or successive contact of intervening bodies with the excited surfaces, as, for instance, by means of pith-balls, pendula, or a blast of air. The former process has been designated by Faraday as disruptive, the latter as convective discharge.

37. The disruptive process being exemplified by lightning, 
the magnificent apparatus of nature by means of which this awful phænomenon is displayed, may be supposed competent to produce convective discharge upon a scale of proportionable magnitude, as exhibited in tornadoes and hurricanes.

38. As bodies oppositely electrified attract each other, a fortiori, attraction must always exist between any bodies suffciently electrified for an electric discharge to take place between them. This law may be illustrated by means of an instrument called Cuthbertson's electrometer. Hence the rising of water within the track of a tornado, and its subsidence on the passage of lightning, as observed by Mr. Allen near the city of Providence, Rhode Island, may be considered as resulting from the alteration of convective with disruptive discharge*.

39. By this observation of Mr. Allen, attraction is shown to have existed between an electrified stratum of air coated by clouds, and the oppositely electrified water of a subjacent river. It is reasonable to infer, that attraction, originating in the same way, operating upon the denser stratum of the atmosphere in the vicinity of the earth by counteracting gravitation, may cause that rarefaction by which houses are burst or unroofed,

* "The most interesting appearance was exhibited when the tornado left the shore, and struck the surface of the adjacent river. Being within a few yards of this spot, I had an opportunity of accurately noting the effects produced on the surface of the water.

" The circle formed by the tornado on the foaming water was about three hundred feet in diameter. Within this circle the water appeared to be in commotion, like that in a huge boiling caldron; and misty vapours, resembling stoam, rapidly arose from the surface, and entering the whirling vortex, at times veiled from sight the centre of the circle, and the lower extremity of the overhanging cone of dark vapour. Amid all the agitation of the water and the air about it, this cone continted unbroken, although it swerved and swung around, with a movement resembling that of the trunk of an elephant whilst that animal is in the act of depressing it to the ground to pick up some minute object. In truth, the tapering form, as well as the vibrating movements of the extremity of this cone of vapour, bore a striking resemblance to those of the trunk of that great animal.

"Whilst passing off" over the water, a distant view of the cloud might have induced the spectator to compare its form to that of a huge umbrella suspended in the hearens, with the column of vapour representing the handle, descending and dipping into the foam of the billows. The waves heaved and swelled whenever the point of this cone passed over them, apparently as if some magical spell were actitig upon them by the effect of enchantment. Twice I noticed a gleam of lightning, or of electric fluid, to dart through the column of vapour, which served as a conductor for it to ascend from the water to the cloud. After the flash the foam of the water seemed immediately to diminish for a moment, as if the discharge of the electric fluid had served to calm the excitement on its agis tated surface." - See Transactions of the American Philosophical So* ciety, vol. vi. 
and an upward current of tremendous force produced. We may also infer that bodies are carried aloft by the joint action of electrical attraction, and the vertical blast which it produces.

40. The effects upon the leaves of trees noticed by me after the tornado of New Brunswick, in 1835, and still more those subsequently observed by Peltier after that of Obatenage in 1839, cannot be explained without supposing them to have been the medium of an electric discharge *.

41. When a convective discharge takes place between a stratum of air in proximity to the earth, and a stratum in the region of the clouds, the greater density and pressure of the lower stratum will determine the current to rush up in a vertical direction.

42. Experience has demonstrated that electricity cannot exist on one side of an electric, without its existence simultaneonsly upon the other side. If the interior of a hollow globular electric be neutral, so will the outside be; but if the interior be either positively or negatively excited, the outside will be found in the one case negative, in the other positive.

43. The atmosphere consists of an electric in the hollow globular form; and as electricity is known to pervade the space within it occupied by earth, the principle in question must also pervade the space beyond that portion of the atmosphere which is sufficiently dense to insulate or to perform the part of an electric.

44. Thus there are three enormous concentric spaces, of which the intermediate one is occupied by an electric, while the innermost one and the outer one are occupied by conductors. The two last-mentioned may be considered as equivalent to two oceans of electricity, of which one may be called the celestial, the other the terrestrial electric ocean.

" In this hasty account I have, with the intention of returning to this portion of the subject, omitted to speak particularly of its effect upon trees. All those which came within the influence of the tornado, presented the same aspect; their sap was vaporized, and their ligneous fibres had become as dry as if kept for forty-eight hours in a furnace heated to ninety degrees above the boiling point. Evidently there was a great mass of vapour instantaneously formed, which could only make its escape by bursting the tree in every direction; and as wood has less cohesion in a horizontal longitudinal than in a transverse direction, these trees were all, throughout one portion of their trunk, cloven into laths. Many trees attest, by their condition, that they served as conductors to continual discharges of electricity, and that the high temperature produced by this passage of the electric fluid, instantly vaporized all the moisture which they contained, and that this instantaneous vaporization burst all the trees open in the direction of their length, until the wood, dried up and split, had become unable to resist the force of the wind which accompanied the tornado." 
45. When either electric ocean is minus, the other must be plus, and at the same time any intermediate stratum of the atmosphere inclosing a stratum of clouds must be charged by induction.

46. Between the concentric strata of air, severally bounding the celestial and terrestrial ocean, there must be an electrical attraction tending to counteract gravitation, and thus to influence the density and pressure of the lower stratum of the atmosphere.

47. The proximities of a stratum of clouds electrified by the celestial ocean, must cause an accumulation of electricity in any portion of the terrestrial surface immediately subjacent; and by counteracting gravitation cause a local diminution of atmospheric pressure, which it is well known is a precursor, and probably a cause of wind and rain.

48. Those common discharges of electricity which take place during hurricanes, may be easily accounted for by supposing that they result from discharges between the celestial and terrestrial electric oceans.

49. Thunder-clouds may owe their charges to the celestial ocean, either by induction or conduction. Auroras may be the consequence of discharges from one part of the atmosphere to another, through the rare conducting medium which is occupied by the celestial ocean; or they may result from discharges from other planets or seas, or from any part of space, however remote. Since, agreeably to Wheatstone's experiments, electricity flies with a velocity not less than that of light, space can create no obstacle to its passage.

LXIV. On the Rotation of a rigid Body round a fixed Point. By James Boотн, Esq., M.A., Principal of, and Professor of Mathematics in Bristol College*.

I. THE problem of the rotation of a rigid body round a 1 fixed point, acted on by no forces, or round its centre of gravity influenced by the force of gravity alone, has been analytically solved by Lagrange, at least so far as to indicate the leading properties of such motion, and to reduce its determination to the calculation of two elliptic functions, one of the first, the other of the third order ; but these analytical formulæ, as has been justly remarked, do not give us any clear idea of the motion during the period of rotation; they enable us to determine the position of the body at the end of a given time, but do not at all assist our conceptions in following the motion of the body during its rotation.

- Communicated by the Author. 